\title{
DE LAS UNIDADES PARALELÍSTICAS EN LAS TRADICIONES ORALES MAYAS ${ }^{1}$
}

\author{
Aurore Monod Becquelun y Cédric Becquey \\ II.PGA, CNRS, Universidad Paris 10
}

Pethaps the most distinctive quality of Mayan literature is that it is all poetry.

Backe. Y Eascevion, 1985: 59

Entre los mayas, el procedimiento llamado "paralelismo" tiene varias denominaciones, usos y sentidos como dyodic sets (Fox, 1974), parallel repetitions (Gossen, 1974a), couplets (Laughlin, 1980), semantic and syntactic parallelism, ${ }^{2}$ couplets structure (Bricker, 1974), gramunatical parallelism (Jakobson, 1973; Norman, 1980). semantic parallelism (Fox, 1975, 1988; Goody, 1979), poetic parallelism (Hanks, 1999: 184), synonymous parallelism (Hull, 2003: 133). Todos estos términos denotan una "combinación de variables e invariables" (Jakobson, 1973: 271), es decir segmentos alternantes dispuestos según limites y latitudes propias de cada lengua y de cada género donde están presentes. Los paralelismos son marcadores tipicos, ya sea de un género entero, de un subgénero, o de tipos de discurso ${ }^{3}$ (Kerbrat-Orecchioni y Traverso, 2004).

Una definición provisional y amplia es la de Burke:

Principles of line organization producing a measured poetics equivalence fin the levels of structure that mark patterning and satisfaction of expectations $|. .$.$| Coordina-$ te ideas are arranged in phrases, sentences and paragraphs that balance one element

\footnotetext{
'Este articulo fue presentado en el VII Congreso Internacional de Mayistas en julio de 2007 en Mérida, Yuc. Es el resultado de un trabajo colectivo realizado por el grupo de investigación "para. Jelismo" en el marco del proyecto "Géographies du sacré: Dynamisme des espaces et des identités mayas" (CNRS, Ministère de la Recherche, Francia). Los datos utilizados fueron recogidos en Bachajón y Sivaci -Chiapas, México (Monod Becquelin, Breton) - Oken - Escobial, Jocotán, Guatemala |Bec: quey)-; Xculoc - Campeche, México (Monod Becquelin). Agradecemos a Miguel Ángel Rodriguez Lizana por su ayuda en la corrección del texto.

"Algunos autores utilizan la expresión "paralelismo sintictico" para designar el co-texto de la frase, idéntico o similar, en el cual se irscribe el contraste de los términos icf. Cassen, 2002, quien opose 'semantic couplets' or nomparallel repetition to 'parallel syntax' or parallel repetitios"), otros lo utilizan para indicar un par cuyos elementos en contraste son gramaticales (aserción/negación; cumplidoincumplido, etcétera).

${ }^{3}$ Los tipos se lacalizan al nivel de las secuencias, no del texto global. Un zénero puede estar constituido de varios tipes: por ejemplo, en un "reza de curación" en Hlachajós, caben maches tipos discursivos: díalogo, narración, plegaria, interrogatorio, pleito, etcétera.
} 
with another of equal importance and similar wording. The repetition of sounds, meanings and structures serve to order, emphasize and point out relations.

(Burke, citado por Darnell, 2003: 823)

Para el mundo mesoamericano y maya en particular, Bricker y Edmonson fundan y establecen el paralelismo en la semántica: "Any two sequential lines should repeat the same idea in synonyms or antonyms $|. .$.$| It is the matching of me-$ anings that matters" (1985: 59). Puede presentarse como pura sinonimia de los enunciados sin repetición de palabras pero con un balanceo semántico perceptible en ambos enunciados (1). La presencia del paralelismo puede ser reconocida a través de elementos comunes a los dos conjuntos paralelisticos $-\mathrm{y}$ en este caso, cada una de las frases que contiene pares está compuesta de un cuadro, cuyo todo o una parte aparece en las dos frases (2) - " "The more tightly they do so, the more formal the style. Sometimes, the parallelism is syntactical, and occasionaly therefore the same suffixes may terminate the lines, resulting in actual sound correspondence or identity, but that does not appear to be required or even necessarily desirable" (ibid.).

(1) de besak ia koa Lilon na kono ma pau bïn na monu

now the tailfeothers'gold strands drop and the binds yellow neckioce folls

(Fox, 1974:75)

(2) 285 laj kahk'otes ta sakal kinal las hice bailar en Ja claridod de la tierra 286 laj kahk'otes ta sakal bahlumilal las fike boilor en la claridod del mondo

(Carnaval de Bachajón, Monod Becquelin, 1973)

El paralelismo ya ha sido bastante estudiado bajo varios aspectos - formales, simbólicos, musicales, pedagógicos-; nuestro interés radica en la función organizadora que desempeña el conjunto de procedimientos descritos por los mayistas. A fin de distinguir los diferentes niveles a los que se aplica, conviene inicialmente señalar su omnipresencia en toda el área mesoamericana y exponer su especificidad "maya". Haremos un recorrido rápido del conjunto de fenómenos que están denotados por la expresión "paralelismo" en la zona que nos ocupa — su presencia en el espacio, en el tiempo y en los medios de expresióny daremos una descripción de las formulas que combinan elementos repetidos y elementos variables. Diferenciaremos el paralelismo de otros fenómenos similares y nos interrogaremos sobre su lugar en la composición de los textos donde aparece (descripción de las unidades, de las disposiciones y de los niveles de organización). Propondremos justificar, aunque sea de manera breve y sintética, la hipótesis según la cual el paralelismo es un modo cognoscitivo de pensar el mundo. Nos preguntaremos finalmente $-y$ se tratará únicamente de una pregun- 
ta- como este doble procedimiento de diferenciación y repetición (similitud / identificación / imitación) se articula con las funciones de los textos, los contextos y las intenciones de los locutores.

\section{Preliminares}

El paralelismo combina elementos repetidos idénticos, casi idénticos o sinónimos, el "co-texto", y elementos contrastivos llamados "pares". En su forma más sencilla, la estructura paralelistica se define por el contraste entre dos términos diferentes anclados en un co-texto idéntico o similarmente repetido.

Los elementos contrastivos presentan múltiples tipos de relaciones retóricas, entre ellas la complementariedad o la antinomia — las figuras más citadas — aunque no sean más que unos ejemplos entre muchos otros.

Como ya lo hemos dicho, el co-texto puede ser idéntico, similar, incrementado o disminuido, o solamente sinónimo. El paralelismo no se restringe a secuencias contiguas sino también a conjuntos enteros y no pocas veces desconectados, jerarquizando niveles más amplios.

La relación que resalta de los elementos contrastivos puede ser neutralizada según el estatuto del par dentro del texto: en este caso, la repetición del binomio pone de relieve el co-texto, el cual, a su vez, asume la progresión del texto.

\section{Extensiones del uso de las formas paralelisticas}

Como bien se sabe, el paralelismo es un rasgo omnipresente en las culturas y lenguas mayas. A continuación daremos algunos ejemplos de la amplitud impresionante del fenomeno en el tiempo y en el espacio, sus varios usos linguiisticos asi como en otros modos de expresión. El inventario de los principales estilos discursivos en la literatura oral y escrita mesoamericana fue hecho por Edmonson y dibuja un largo y detallado panorama de los procedimientos y géneros (Edmonson, 1986).

\subsection{Extensión en el espacio}

Es posible constatar que el paralelismo, presente en las lenguas y culturas del mundo, se despliega en formas variadas, más o menos desarrolladas y apremiantes; que no implica una tradición oral o al contrario, escrita, sin embargo es conveniente reconocer que las tradiciones escritas donde se manifiesta, proceden claramente de tradición oral. 


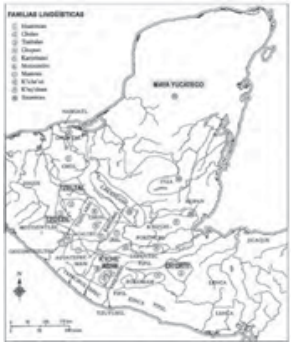

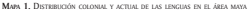
[Seguin Coe, en Sharer 2006: 27). Los numeros se refieren a las familias linguisticas, los nombres en maviscula a los idiomas individuales y a los grupos étnicos. Las partes en gris son las lenguas utilizadas en los ejemplos

A continuación damos algunos ejemplos de pares en estructuras paralelas tanto en textos escritos como en transcripciones de tradición oral contemporánea, y de diferentes lenguas: ${ }^{4}$

\footnotetext{
${ }^{4}$ Las partes idénticas o similares no se resaltan y las partes contrastivas estain en negritas.
} 
(3) ka'h kint'anajoob bakan ti' mosono'ob ik'o'ob

tī xkan p'el ik'o'ob

tî $x$ 'ojo moson

tí sak moson

tỉ xk'aan mosoon

ka'h kint'anajo'ob habiarín ${ }^{5}$

a los remolinos

a les vientos

a los cuatro vientos

al ojo del remolins

al remalino blanco

al remolino asterillo

hablarín

(Ritual de las primicias, Monod Becquelin, 1988, recogido en Xculoc)

\section{Tzeltal}

(4) bin k'an kutik, tatike

sakix

que vames hacer, podres.

bawitsaj wan te mahtantesel

yo está amaneciendo

te oboltesel

ts'akal wan jayeb ora

ts'akal wan jayeb ihk'al

la wan behentayix ora

la wan behentayix k'ahk'al

estamos a punto de hacer sus ofrendas

su contribución

mechas hores se fian completadas

muchas ascuridades se han completados

han cominado las horas

ta yutil swokol

han cominodo las dias

ta yutil ame'ba'al

en sir sufrimiento

en su soiedod

ma' me laj ach'ojon

to me has rechozado

ma' me laj asuhton

no me hos echodo

la me jtahbey yorail

yo Jo alcancé su hora

la me jtahbey skahk'alel

yo to alcancé se día

la me jkuch jun yal ik

yo aguanté fos vientas

la me jkuch jun yal ahk'abal

yo aguanté la noche

la me jkuch jun yal serena

yo aguanté Ja humedod frocio de Ja mañana)

te muk'ul te'etik

los grandes érboles

te muk'ul ch'ajanetik.

los grandes bejuces

(Primera entrada de pedida de la novia, Breton, 1992, recogida en Bachajóo)

\section{Ch'orti'}

(5) ch'a's e holcthan b'aki che Se encuentro el mal ojo de los huesos ch'a's e holchan harari che se encuentro el mal ojo de los carrizes

\footnotetext{
${ }^{5}$ Las traducciones de las citas son las de los informantes, saho mención explicita.
} 
ch'a'f a'si tamas enyax rio che tamar ensak rio che

a'asi tamar enyax playa ensak plays se encuentro jugando en el rio verde

et ef róbianco

juega en la ribera verde

lo ribera blanica

ch'a'f takar ususedyente yr uyok che se encuentra com el susto de sirs pies takar ususedyente'yr ukab' che con el sursto de sus mentos

(Rezo de curación, Becquey, 2007, recogido en Oken)

Tzotzil

(6) K'usi ti nopbile, K'usi tí p'isbile? Mi nat to stek'el. Mi nat to xanel, Lanichinal baike, Lanichimal satike? Nichimal jtot, Nichimal kajval. Ja' me yo ke ech'el, fa' me yo jti' ech'el.
Que ha side decidido, quie ha sido medido? Esté todovía lergo su camino. Estó todavía lorgo su viaje, sus lindos rastros Sirs lindos ojos? belleza de mi podre, belleza de mi ser̂or por eso se wa mi tardía boca. por cso se wan mis tordios labios.

"The Man of the House Venerates the Candles", Laughlin, 1980: 208)

Existe una gran constancia no sólo en las formas escogidas - una parte variante, otra invariante- sino también en los términos pareados.

Se encuentran pares tales como "pies/manos" en muchas lenguas de las diferentes familias; a veces los pares presentan las mismas raices, $o$, en otros casos. raices diferentes pero con sentidos identicos o comparables. ${ }^{6}$

Raices idénticas:

$(7)$

$\begin{array}{lll}\text { Ch'orti } & \text { ok } / \mathrm{k}^{\prime} \mathrm{ab} & \text { pies / manos } \\ \text { Tzeltal } & \text { ok } / \mathrm{k}^{\prime} \mathrm{ab} & \text { pies / manos } \\ \text { Tzotzil } & \text { ok } / \mathrm{k}^{\prime} \mathrm{ob} & \text { pies / manos } \\ \text { Lxil } & \text { oj } / q^{\prime} \mathrm{sb} & \text { pies imanos }\end{array}$

Raices diferentes con sentido similar:

\footnotetext{
"Los irmentarios han sido efectuados a partir de diferentes tipos de textus: diccionarios, glosas espontaineas y elicitaciones, discursos rituales.
} 
(8) Achi al/ k'ajol hijo de mujer / hijo de hombure

Tzeltal al / nich'an hijo de mujer / hijo de hombotre

$\begin{array}{lll}\text { Achi } & \text { qij/dyora } & \text { dia/momento } \\ \text { Tzeltal } & \text { kahk al/ora } & \text { día/momento } \\ \text { Tzotzil } & \text { diya/ora } & \text { dia/momento }\end{array}$

No raramente los pares léxicos/lexicales son más especificos de una región, una lengua, un ritual:

(9) Achil q'uq' / raxon quetzal/piomes vendes la ecologia de las tierras altas y el arte de indumentaria

\begin{tabular}{|c|c|c|c|}
\hline Achi & $q^{\prime} \operatorname{an} / \operatorname{rax}$ & amarillo / verde & \\
\hline Achí & $q^{\prime} a n / s a q$ & amerillo / blanco & cada lengua escoge \\
\hline Tzeltal & sak / ihk & Blanco / negro & su contraste de color \\
\hline thorti & yax $/ \mathrm{sak}$ & verde / blanco & \\
\hline Tzelcal & aw / ts'uts'up & griter / silbor & $\begin{array}{l}\text { al ritual de cambio de varas } \\
\text { del carnơval de Bachajón }\end{array}$ \\
\hline
\end{tabular}

\subsection{Extensión en el tiemupo}

Tenemos numerosos indicios del uso det paralelismo desde los textos epigráficos hasta la literatura contemporánea (Lounsbury, 1980; Lacadena, en prensa; Hull, 2002; Bricker, s.f.). He aqui un ejemplo interesante, ya que se trata de un leitmotiv muy presente en la tradición oral que involucra conceptos relativos al tiempo.?

\footnotetext{
${ }^{5}$ En la predicatoria de Chan Santa Cruz citado por Bricker se encuentra $t \omega$ mérn K'wch $\mathrm{f} u$ borahil $t u$ ba'abil "because it has arrined in the hour, in the year" (Bricker, 1974: 385; en tzotzill, los pares unen los dias con los meses te lajicheb 'we, to lajcheb k'ak' aile "en doce meses, en doce dias", las fiestas con los rituales ta smuk'ta k'in, to somuk'ta paskun "en las fiestas en el ritual", el momento - en el

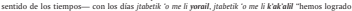
el tiempo justo, hemes logrado los dias adecuados"; en tzeltal se encuentra también los pares ora / habil, oro / Kahk'al y muchos otros. Ora, en estos contextos, no significa 'hora", sine mis bien "tiempo adecuado", "tiempo previsto"; en este sentido, esti frecuentemente asociado con sbahbial "del atigen, primero", y k'in con zu tsilal bahluwilal, "el bienestar deł mando".
} 


\section{Epigrafia maya}

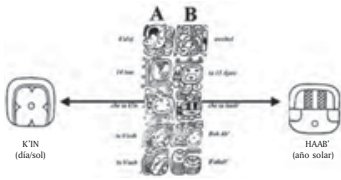

K'alaj uwohol

14 tuun

ta 13 Ajaw

che ta k'in

che ta hab'

tu b'aah Boh Ab

tu b'aah B'akab' fue atodo sas glifos

c' ẩo 74

ent el Kotun 13 Ajow

fue dicho en el dío

fue dicho en el año

su retrato Boh Aab.

su retrato Brokab'

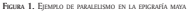

(Vhso Kerr $\$ 017$ dibujado por K. Hull, 2002-9)

En El rituol de los Bacabes (yucateco colonial) también se encuentra este tipo de formas parecidas:

\section{Maya yucateco colonial}

(10) Zam tun bacin in chucech cen a na,

cen a yum.

Cech Zacal Can Tippte be chee, cech Chacal Can Tippte be chee.

Max bin be chee cech ocic kin be chee ahic cabe chee?

Cech u coil al be chee, u coil chab be chee?
De repente he agarro Yo quiven sty su modre Yo quien soy su padre.

Oh Usted enfermedod intestinal serpiente bionco oh Usted enfermedod intestinal serpiente rojo.

Oh? Quien es usted of creptiscilio of alba?

Oh es Usted el thĩo de la rabia. la creación de ia rabia?

CFor Snake Intestinal Paints in a Person's Stounach; Very Good", Bolles y Bolles, 1996, Apéndicel 
Las "conciones" redactadas por un clérigo castellano, el padre Manuel Diez, demuestran un conocimiento agudo de la formalización en pares; podemos verlo en este inicio de prédica en la celebración del día de Santa Rosa:

\section{Tzeltal colonial}

(1) Latocal avabijc ghalal quich nichnab ghun buhtzan cop yutun nax znael yuun nax yabiel zghalal zpatzoghel qmetic $S^{*}$ cuxulto ta balumilaye.

tento proferidb, queridos hijos, una palabra bouito para que sea rementorada para que sea escuchoda Las fiechos maravillosos de nuestra madre Cuando vivia todavia en el mundo.

C'Zghalal zpatzoghel qmetic S.Rosa cuxulto ta balumilar' [1675], Rux, 1989: 360)

Sin embargo, el paralelismo está limitado al uso de términos pareados en la imitación de los géneros rituales, sin contribuir en la organización del texto a niveles más amplios (Monod Becquelin, 1995: 89-90).

Achi de Rabinal

(12) 45 katel uloq worom ajaw 46 [k]aqoun ajaw

47 xa k'u xere chi nabe wae ma wi

48 kaønuk'iso uch'ayik uwixal

49

50 ajaw chakachib

51 tzamanib

52 kawuq rabinal

53 ka@cha Ku fi nutzij ch(i) uwach kaj 54

\section{ukutamil}

chlit uwach ulew
iWes a sefir, soberano agujerado saberono perforado!

No seras el primero cuyas roices

cuyo tranco acabo

$\rightarrow$ rey de las chakachio de los tzontenth

Kawing Robinal.

dice mi palobre a la faz del cielo

a la foz de la tierra.

(Rabinal Achi, Breton, 1999. 144-145)

La poesía contemporánea también lo usa como uno de los instrumentos para la belleza de las imágenes y el vigor de las sonoridades asociadas.

\section{Maya yucateco}
(13) Tele
[...
E gofio
tu ch'ich'ikik u nek'il in páakat
[...]
inserta en la pupila de mis ojos
yéetel u ch'ich'iltúnil u moch';
los guijarros de su garra; 
tu t'ot'och'ik xan u neek'il in páakat picotea la pupila de mis ojos

yéetel u k'an lóche'il u koj.

con el amarillento garfio de su pico.

(to paiaht yótel juntul $t$ 't, Mirada can un gallo, Cuevas Cab, 2007: 7)

\subsection{Usos transgenéricos del paralelisno}

El paralelismo se manifiesta como procedimiento discursivo denso en los géneros rituales, pero también en las situaciones más cotidianas de interacción (de lo doméstico a lo intercomunitario) y en todas las ocasiones de ritualización, de emoción, ${ }^{8}$ de interacción con los ancestros, autoridades o entidades sobrenaturales asi como, de una manera más moderada, en el uso cotidiano (Haviland, 1992; Monod Becquelin, 2007). ${ }^{*}$ La extensión transgenèrica de este fenómeno se ve ilustrada en varias situaciones: defensas tzotziles, cambios de cargo tzeltales, plegarias ch'orti'; en yucateco está presente en proverbios, rezos, profecias, narrativas históricas, adivinanzas (Bricker, 1974: 381); se encuentra también en "mitos" y cuentos. Más aún, el paralelismo surge también en el hablar cotidiano para precisar el concepto utilizado.

Encontramos un ejemplo de este uso en la elicitación del valor simbólico del color de la luna y de la llegada de la temporada de lluvias, donde, de repente, surge el paralelismo:

\section{Ch'orti'}

(14) e katu' watat chakopen inyajrer uwirna't la luna oparece muy roja en su aporiencia, uwarar en su refiejo.

(Hull, 2000: 4)

En un contexto de elicitación de un texto de cura ritual, a la pregunta sobre cuál es el sentido de la palabra lat'ahnir (catatonia), el curandero responde terminando su explicación con paralelos:

(15) - Llat'alunir?

-ch'a'r takar ulat'ahnir uyok
Elat'ahnir?

el (espirin de la enfermedad) se encuentra con Ja catatania (lat'ahnir) de sus pies.

Está destragado, está destraga|do|. Esté (el enfermo) destragado, esté destrogo|doj.

\footnotetext{
'En contexto maya, experimentar emoción puede llevar al uso de formas paralelisticas en el discurso.

"Hull distingue los géneros rituales de les gèneros narratiosos los gèneros rituales se caracterizan por el empleo de formas arcaicas - tavta léxicas como gramaticales-y también de instrumentos comunes a los dos tipos de textos pero en proporciones sensiblemente diferentes: paralelismo, prés. tamos al español, figuras retóricas, elipsis, onnamentación, etc- (Hull, 2003: 81).
} 
la persona no come

[...]

ma'chi awe

ma'chi uyuch'i e pa la persona no come

|... |

No conte

No bebe la carnido

(Entrevista en Oken, Becquey. 2007)

Tzotzil (7 de agosto 1812 )

Tenemos otro buen ejemplo de conocimiento de los mecanismos creativos de paralelos en los versos citados por Bricker en los cuales un padre bilingie (castellano y tzotzil) traduce y reescribe en paralelos una proclama del rey de España a los indios de Chiapas para que desconfien de Napoleón:

(16) 38 Ytal ta yan slecó́g osil

39 Jun coló huinic

Hun mu ibeiluc tzameshuaneg

40 Napoleon sbiil.

41 Spasog sbá lolohuaneg

Spasog sbá gnoxol wise de atra

de ojeno tierra

un hombre mabo

un esesino trasdor

Nopodeón es su nombre

se nolvio engatador

se volvíu vecino

("Proclamación del rey de Espania en Cidiz a los indios de Chiapas", Bricker, 1974: 379-80)

\subsection{Uso con otros medios de expresion}

En efecto, el paralelismo no se confina en la lengua; en otros modos de expresión encontramos - tanto en la iconologia, como probablemente en la disposición de elementos arquitectónicos- ejemplos de simetria compleja que remiten al paralelismo. Tomaremos el caso del monstruo terrestre.

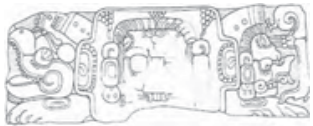

Focuan 2. Monstruo Kuwox [CPN tot dibujada por B. Fashi 
El monstruo Kawak de dos cabezas es un icono que expresa los dos aspectos contrastados de la tierra. A la izquierda, la cabeza de un sapo, simbolo de la tierra húmeda y fértil; a la derecha, una máscara esquelética, simbolo de la tierra ärida (segun Baudez).

Si admitimos la asociación con la tierra de estas dos representaciones - sapo y máscara esquelética - podemos concebirlas a la vez como equivalentes y contrastadas. Las caracteristicas pertinentes que oponen cada una de estas dos cabezas forman el par húmedo / árido. El dispositivo paralelistico flanquea la máscara central donde aparecen los elementos del glifo "kawak" |T528v| siempre presente en las representaciones del monstruo terrestre.

Aqui se ven numerosas relaciones retóricas sucesivas o simultáneas: la sinécdoque de un sapo (cabeza), una metáfora de la muerte (esqueleto) y una metonimia (cráneo). Esta oposición forma parte de un sistema que abarca un paradigma de substituciones para identificar al monstruo terrestre: la serpiente carnosa vs. la serpiente sin carne, el cocodrilo vs, el jaguar, etc. Es efectivamente la presencia de un verdadero sistema de oposiciones lo que hace de esta figura hibrida un ejemplo de paralelismo.

La generalidad y la extensión de este proceso nos invitan entonces a proponer una tipología detallada.

\section{Descripción}

Pairing of ideas and phrases - semantic and syntactic couplets - is as common to tzotzil language use as com is to the tzotzil diet.

Gassex, 2002: xtvil

La cita de Gossen vale no sólo para los tzotziles sino también para todos los pueblos mayas, aunque el paralelismo se usa en cada grupo con sus rasgos caracteristicos. Hay que diferenciar dos conceptos próximos at paralelismo: el difrasismo y la repetición. El entendimiento del difrasismo es crucial para seguir la continuidad del paralelismo al difrasismo y a la composición a través de procesos de estereotipificación. Por otro lado, se debe distinguir el paralelismo de la repetición - herramienta retórica importante- que carece de la parte variable, en oposición al paralelismo. La transición es muy leve entre los tres procedimientos pero útil para entender la organización discursiva. Cada uno de estos instrumentos estilisticos forma parte de los recursos utilizados en la construcción del texto. Ahora bien, no tienen todos el mismo estatuto cognoscitivo, como lo veremos más abajo. 


\subsection{Figures contiguas}

\subsubsection{Difrasismo}

El difrasismo consiste en la asociación (estable) de dos lexemas, lo que resulta ser una manera de construir un significado nuevo expresando una idea única, en la cual el sentido final no es co-extensivo a la suma de los sentidos de sus dos componentes (Garibay, 1953). Es efectivamente en la literatura nahuat clásica y moderna que se ha analizado precisa y detallamente el difrasismo (Launey, 1992). El difrasismo tiene mucho que ver con el paralelismo; los dos proceden de las asociaciones más importantes y más frecuentes en la cultura. ${ }^{10}$

lacadena lo define asi: "una figura semántica compuesta de dos elementos que constituyen una unidad estable de significado metafórico" (Lacadena, en prensa). Además de subrayar la estabilidad del conjunto, se hace hincapié en la yuxtaposición de dos (o aun tres) lexemas cuyo significado no se construye a través de la suma de sus partes, sino que remite a un tercer significado como en tzicuehua "astillar" + tlapani "romper" .-.> "nacer"; o in atl "agua" + in metlatl "metate" $\rightarrow$ "mujer" (Montes de Oca Vega, 1997).

\section{A) Epigrafia}

Bien se conocen los ejemplos mayas del difrasismo en la escritura glifica: pohp / Iz'am: "estera / trono" $\rightarrow$ "poder político", kab'/ch'en: "tierra / cueva" $\rightarrow$ "ciudad, territorio". Stuart (2003) argumenta sobre la existencia de este fenómeno: ${ }^{1 t}$
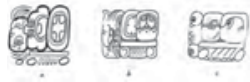

\begin{tabular}{|c|c|c|c|c|c|c|c|}
\hline YAX & verdejazul & + & $\begin{array}{l}\mathrm{X}^{\prime} \mathrm{AN} \\
\mathrm{AHK}^{\prime} \mathrm{AB}\end{array}$ & $\begin{array}{l}\text { amerillo } \\
\text { noche }\end{array}$ & $=$ & K & W.IR \\
\hline HAN & Cielo & + & KAB' & tierra & & & \\
\hline
\end{tabular}

Ficuer 3. Vaunsies ercaificas del. Denasisso TZ'AK IDibujadas por linda Schele, Stuart, 2003: 21

\footnotetext{
"Bricker y Edmonson encadenan obviamente el difrasismo a la forma paralelistica: The very formal contexts in which they are employed may lend such expressions an additional and esoteric semantic force, endowing the coupled concepts with a third and dialectically derivative meaning " (1985: 60).

11 "It is important to stress that the two elements are not read as separate signs, but instead stand together as a single unit representing the word TZ'AK" (Stuart, 2003: 1).
} 
Estos tres bloques de glifos ilustran tres maneras diferentes de escribir la palabra uts'akah "cumple/cumpliō". Aquí, la raiz TZ'AK "cumplir" puede ser representada por la asociación azul-verde/amarillo (a), dia/noche (b) y cielovtierra (c). Los dos ültimos casos elaboran el concepto de completud extirpado de la asociación de los dos términos, mientras que el caso (a) es una figura más compleja de entender.

\section{B) Textos modemos (tzeltal)}

Difrasismos parecidos a las figuras canónicas del mundo náhuatl, existen en las lenguas mayas, tales como en tzeltal me'il.tatiletik "ancestros" (madre.padre). En los discursos rituales se encuentran muchos pares que, con el tiempo, se convierten en difrasismos perdiendo vitalidad y probablemente sustituidos en productividad por pares más actualizados, tal como lo muestra el siguiente ejemplo. traducido por un locutor tzeltal:

(17) 160 ay to ta amahtantesel 161 ay to ta awoboltesel

$$
\begin{aligned}
& \text { mahtan }+ \text { obol } \\
& \text { ofrende }+ \text { contribucion }
\end{aligned}
$$

185 ay to te mahtan.oboltesel ${ }^{12}$

186 ay to te limosna.obligasyon
Hay todonía ta ofrendia

Hay todovía tu contribuciein

$$
\begin{aligned}
& =\text { mahtan.obol } \\
& =\text { suffimiento }
\end{aligned}
$$

Hay todovía ef sufrimiento

Hay todovía la prinociónt

(Primera entrada de la pedida de novia, Breton, 1992, recogido en Bachajón)

$A$ veces, se vuelven verdaderos compuestos - con una única marca morfológica para los dos elementos- hasta perder la etimología de los componentes como en cham,balam "animales" (serpiente.jaguar). ${ }^{13}$

\subsubsection{Repetición}

En general, se reparten las funciones de la repetición entre dos polos:

\footnotetext{
${ }^{12}$ El difrasismo mantiene la posibilidad de llevar flexiones y derivaciones, lo que lo distingue de las frases idiomáticas (linguiscic freczes).

" Cuando es "inventado" y difundido, el difracismo es 'fresh, vigorous and dynamic" lasegún León-Partilla, citado por Hull, 2003: 137) su uso repetido y banalizado lo vuelve monoléxemico y menos creativo.
} 
- ayudar a la comprensión y a la consolidación del mensaje (con todos los fenómenos de redundancia y de defensa ante el "ruido"):

- producir emoción, alertar al interlocutor, reanimar su atención, insistir en la cohesión del texto.

Deborah Tannen (1989) realizó un estudio con respecto a la relación entre paralelismo y repetición conversacional y proporcionó un resumen interesante de las investigaciones desde diversos puntos de vista: psicologia, antropologia, linguística. Ella señala que la repetición es un fenómeno general de la interlocución, a cada nivel del lenguaje: producción, comprensión, interacción (para los mayas, véase Brody, 1986). Comparando los resultados con los de los estudios que consideran la dimensión poética de la repetición —muchas veces confundida con el paralelismo- concluye citando un conjunto de funciones compartidas, señaladas por Grimes como "linkage, expansion, continuation, information focus, emotional involvment" (Grimes, 1972: 576).

Volvemos a nuestro punto de partida: la estructura textual, en donde paralelismo y repetición requieren comparación. La repetición, muy estudiada desde el punto de vista de la estructura de los cuentos europeos, ha sido pocas veces comparada con el paralelismo. Éste ha sido analizado como figura retórica más que en tèrminos de un proceso organizador de textos. El paralelismo no se presenta como repetición casual, fortuita, sino como repetición organizadora de "algo mayor": estos elementos que se retoman construyen un sistema que comprende varios tipos de unidades y varios niveles que solicitan sin cesar la memoria.

Las dos herramientas están presentes en la literatura y tradición oral maya con la diferencia de naturaleza que proponemos como hipótesis: la repetición permanece del lado del enunciador en el orden de lo subjetivo, mientras que el paralelismo, al mismo tiempo organiza el discurso del enunciador y la memoria colectiva de los oyentes. El paralelismo tiene un rol importante en la tarea de transmisión del conocimiento junto con las formas adecuadas con que se elabora este conocimiento. Jill Brody insiste sobre la función preponderante de "reinforcement of social solidarity" (Brody, 1986: 259) que adquieren ta repeticion y el paralelismo en la comunidad maya tojolabal. En el aprendizaje, la comprensión es suministrada por la experiencia emocional en obra durante la interacción; Halliday señala el hecho de que la repetición es un modo de resaltar la postura, la posición del enunciador (Halliday, 1967). En efecto, hemos comprobado en varias versiones de los discursos rituales del carnaval que paralelismo y emoción se desplieguan y se confirman de manera mutua (Monod Becquelin y Breton, 2002): se justifica ampliamente la cita de Becker: "The actual a-priori of any language event - the real deep structure - is an accumulation of remembered prior texts $[\ldots \mid$ thus our real language competence is access, via memory, to this accumulation of prior text" (Becker, 1984: 435), aun más cuando el texto se presenta con estilos formales. 


\subsection{Paralelismo}

El paralelismo se encuentra en cada nivel de la lengua. Todas las unidades linguiisticas (prosodia, morfemas, palabras, sintagmas, frases, enunciados) pueden entrar en los juegos de contraste. Bright nota, en los procedimientos paralelisticos, "high level prosodic features such as overall rhythme, patern of loudness, duration of pauses (and many other prosodic and phonetic features), on one hand, and hierarchical patterns of morphosyntaxtic and semantic organisation in the other hand" (Bright, 1990: 437-438). Se darán algunos breves ejemplos.

\subsubsection{Prosodia}

Constatamos en la figura 4 que la última vocal le:| del mi-par - uno de los elementos del par- (teh/umale), la que lleva el acento, dura 6 veces más que las otras. Comenzamos por este parámetro porque es frecuentemente mencionado pero raramente medido con precisión. Por lo tanto, se asocia al paralelismo con figuras prosódicas especificas; algunos rasgos varian según los locutores, los contextos y los dialectos, otros entre comunidades: melodia, tonalidad, acentuación, ritmo y pausa dan sus identidades a los usos del paralelismo.

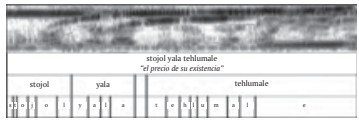

Fianer 4. Segmextanones de ux verso

(Camaval de Bachajón, Monod Beccaelin, 1992)

El cuadro 1 nos permite hacer las observaciones siguientes:

Cuadno 1. Dusacxín de las vocales de la livea

\begin{tabular}{|l|c|}
\hline & Dunucoin (SECUNoo) \\
\cline { 2 - 2 } Vocales no acentuadas (media) & 0.047 \\
Vocales acentuadas en posición mediana (media) & 0.088 \\
Vocales no finales (media) & 0.067 \\
Vocal final & 0.400 \\
\hline
\end{tabular}


La prosodia es un fenómeno sumamente complejo, que aún no ha sido estudiado plenamente, sin embargo, sabemos que algunos de sus parámetros, como la velocidad, dependen de la habilidad del locutor, del momento del performance y de los participantes del diálogo.

A continuación, las figuras 5 y 6 representan exactamente 10 segundos de grabación ilustrando una cadencia rápida y otra lenta dentro del mismo performence.

Esta primera figura ilustra un performance rápido con un promedio ritmico de un verso por segundo:

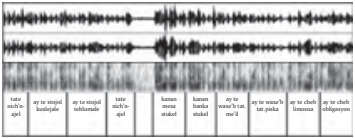

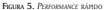

(Carnawal de Bachajón, Monod Becçaelin, 1992)

En la figura siguiente podemos apreciar un performance lento donde todos los versos están entrecortados de pausas, lo que resulta en una cadencia con el promedio de un verso cada dos segundos:

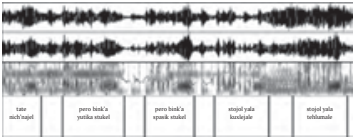

Ficura 6. Pexposuna LeNTD

(Carnawal de Bachajón, Monod Becçaelin, 1992) 


\subsubsection{Paralelismo morfosintáctico y semaintico}

El contraste entre los términos puestos en paralelo se puede producir al nivel de una raiz o de un morfema gramatical (que denota tiempo, aspecto, modo, persona, o negación, en los casos más comunes), de una palabra o de un sintagma. Además, los tipos de contrastes pueden combinarse y no se excluye ninguna categoria.

A continuación damos algunos ejemplos de pares de raices, de aspecto, del primer elemento de un compuesto y de sintagmas.

\section{- Raices}

Tzeltal

(18) 239 Mahtanijat tal

240 O'bolijat tal

697 La me smahtantesat tal kajwal

698 La me yoboltesat tal kajwal
Has venido a ofrecer

Has ventido a entregar

te ofrecerún en (a) if señor

te entregeran en (a) ti serior

(Discurso del ilutive -especialista ritual-, Monod Becquelin, 1974, recogido en Snaciil

- Morfemas gramaticales

Maya yucateco

(19) wa tuch ten loobe' a st me surede algo malo wa / $\mathrm{k}$ (hipotético /

habitual)

kuyíuchu te'ex loob le sirede algo malo a ustedes te'ex / ten (ustedes / me) wa ma' tuch ten loobe' si no me sucede nada maís wa ma' ।

mix ba'a (si no / nada)

mix ba'a kuyutuchu te'ex noda malo le sucede a ustedes

(Palabras de Ki'ichlkelem Taata Juan de la Cruz Vapoarsky, 1999)

Tzeltal

(20) La kak'beytikix skahkahil Ya kak'beyix sk'ahk'alil
Ya fe dimos su tiempo Yo le danos su tiempo la $\int$ ya (perfectivo /

imperfectivol

(Camaval de Bachajón, Monod Becquelin, 1973) 


\section{- Elemento de compuestos}

El contraste puede encontrarse también en una parte de la palabra, por ejemplo uno de dos elementos de un compuesto.

Tzeltal

(21) 747 Te ma' la jts'aktik sme'elo'tan A veces quedames huérfanos

748 Te ma' la jts'aktik sjalal.o'tan A veces quedames alejodos

(Carnaval de Bachajón, Monod Becquelin, 1973)

- Sintagma

Tzeltal

(22) 88 Ta sts'akel spatan kajwal AN cumplir su obra del señor

89 Ta sp'ijubtesel snich'an Para civilizar a sus hijos

[MT. al causar quie se vuelvan sabios sus hijos]

666 Ta jun yal yutil ok'om Dentro de un pequeño abrig̀

667 Ta jun yal sme'ba'al snah dentro de un pequeño humbilde fiogor

(Discurso del ilutive -especialista ritual-, Monod Becquelin, 1974, recogido en Snaci)

El ejemplo (23) y el cuadro 2 muestran la intrincación de combinaciones posibles, que pueden ser mucho más numerosas, como si el recitante se divirtiera multiplicando los juegos de espejos y de memoria.

(23) 140 ya $\mathrm{x}$ ochotik ta yohlil $\mathrm{k}^{\prime} \mathrm{LN}$ bak entramos/entrarentes en el medio del freso tierno

141 ya $x$ ochotik ta yohla rease bak'et entramosientraremies en el medio de fa carne

142 wayalon ta yohlil bak aplastodo

143 wayalon ta yohlil pechel baket estoy dormido en el medilo der hueso estoy dormido en el medio de la carne aplastodia

144 beyom chawajon soy bilis cominante

145 beyom jul.bakot soy rewine cattinionte

$146 \overline{0 \times}$ hul jo'on ta chi entrado yo mismo en la vene

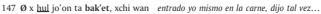

['Mais jai transmis fesperance", Bretos et Monod Beccpuelin, 1989) 


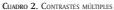

\begin{tabular}{|c|c|c|c|c|c|c|}
\hline & $\begin{array}{l}\text { Ceasuticici } \\
\text { aspecto }\end{array}$ & $\begin{array}{l}\text { Lricon } \\
\text { verbo }\end{array}$ & & $\begin{array}{l}\text { Consuitich } \\
\text { persona }\end{array}$ & $\begin{array}{l}\text { Lrsan } \\
\text { adjetivo }\end{array}$ & $\begin{array}{l}\text { Lraco } \\
\text { sustanstionos } \\
\text { \{cuerpo| }\end{array}$ \\
\hline 140 & $\begin{array}{cc}y a x & \text { "imperfection } \\
& \text { intransition }\end{array}$ & och "entrar" & otik & $\begin{array}{l}\text { "j persona } \\
\text { absalutiva plaral" }\end{array}$ & I'un "biemn" & baik "hweso" \\
\hline 141 & $\begin{array}{cc}y a x & \text { "imperfectivo } \\
& \text { intransitiv" }\end{array}$ & och "entrar" & otak & $\begin{array}{l}\text { I" personata } \\
\text { absolutim pumar" }\end{array}$ & pechel "apinstado" & balket "carme" \\
\hline 142 & & way "dormir" & on & $\begin{array}{l}\text { "I persona } \\
\text { absolutiva singwar" }\end{array}$ & $=$ & balk "hweso" \\
\hline 143 & & way " "dormir" & on & $\begin{array}{l}\text { "I" persona } \\
\text { absolutiva singwhar" }\end{array}$ & pechel "apinstedo" & bail et "carme" \\
\hline 144 & & be "camintar" & on & $\begin{array}{l}\text { "I" persona } \\
\text { absolutiva singwar" }\end{array}$ & & chaiwaij "botis" \\
\hline 145 & & be "raminar" & on & $\begin{array}{l}\text { "14 persona } \\
\text { absolutiva singmbar" }\end{array}$ & & pul.bak "newma" \\
\hline 146 & $\begin{array}{c}\sigma^{1+} \mathbf{x} \text { "perfectivo } \\
\text { intramitiv" }\end{array}$ & hul "Wegror" & jo'on & $\begin{array}{l}\text { "pronombre } \\
\text { independiente } \\
\text { de ia } \mathrm{j}^{4} \text { persona } \\
\text { singular" }\end{array}$ & & "vema" \\
\hline 147 & $\begin{array}{ll}\times & \text { "perfection } \\
& \text { intransitiv" }\end{array}$ & hul "negar" & pón & 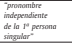 & & bail et "carne" \\
\hline
\end{tabular}

\subsubsection{El co-texto: elipses y aumentos en el co-texto}

Hemos definido provisional y arbitrariamente el co-texto como el conjunto de los elementos que permiten identificar los pares (parte variante del enunciado) en contraste con los elementos fijos, idénticos o similares que cercan $\longrightarrow$ o preceden o siguen- los pares; los limites del co-texto son estrictamente co-extensivos a la parte adyacente, sin tomar en consideración la completud o la incompletud del enunciado.

La elipsis de elementos del co-texto es muy frecuente, con supresiones que pueden abarcar desde una palabra hasta casi todo el co-texto.

\section{Tzeltal}

(24) 283 yak ta tulanijel yal yok ${ }^{15}$ 284 -- ta tulanijel yal sk'ab estain wolviendo fuertes a sirs pies

están viviendo fuertes a sirs manes

(Carnaval de Bachajón, Monod Becquelin, 1992)

\footnotetext{
${ }^{14}$ Ausencia del aspecto imperfectino.

15 L letra negrita senala los elementos del co-texto.
} 
(25) Tey me laj yahtay jayeb ora -jayeb k'ahk'al alle hicieran lo cuenta de cuanto tiempo.

de cuantos dias

(Camaval de Bachajón, Monod Becquelin, 1992)

(26) 97 xochotik ta Kop ta yohlil chi Entrames para hobler en el medio de lo vena 98 -........ bak'et de la carne

(Rezo para huesos quebrados, Breton, 1974, recogido en Bachajōa)

Ch'orti'

(27) Aasi taka uyatravesir ubolchanir ukolera Ellos juegon cant su fuerza inhibidoro del calor de su cólero yustisirantir ukolera é suspiro de su cólera usakb'urichir ukolera el simple calar de su cólera

(Hull, 2002: 6)

El fenómeno inverso, de expansión interna del co-texto o del par existe tambièn, asociado a veces con la elipsis.

Tzeltal

(28) 81 ya j-tuptik lok'el te biyon chi

82 oxlajuneb ya j-tuptik

apago para que salga la enfermedad de la vena lok'el te yokom chi trece veces opago para que saiga la enfermedod de is venta $[\ldots]$

318 ink'-bal ts'anabul

Listerd negro el esplendor

319 sak-bal ts'anabul estard bianco el espiendor de tu corazón aw-o'tan y-o'tik abora?

(Rezo de curación, Monod Becquelin, 1992, recogido en Bachajón)

\subsubsection{Más sobre el co-texto y el contexto de los pares}

Debido a la importante plasticidad de contrastes que pueden aplicarse en todos los lugares del texto, los distintos co-textos modifican el sentido del par al cual son asociados.

A continuación daremos un ejemplo simple de varios co-textos del par a'tel "cargo, trabajo, cargo" / patan "obra, contribución" en un texto de cambio de varas en Bachajón, con la traducción provista por un locutor tzeltal (entre corchetes, agregamos la traducción literal cuando sea necesario). 
Tzeltal

(29) 87 Ta sts'akel ya'tel kajwal

Ta sts'akel spatan kajwal

118 Te sjal|al] a'tel

Te sjal[al] patan

283 T'ujbil yutsil a'tel

T'ujbil yutsil patani

291 Ts'akal me a'tejon

Ts'akal me patanijon

299 Arejon me

Patanijon me

485 Ya kilbeytik ya'tel Ya kilbeytik spatan

492 Ya sts'akay sjalal atrel Ya sts'akay sjalal patan
En fos tiempos del cargo de auestro señor [al cumplif el cargo del senoc| ${ }^{16}$ Al cumplir la obra del señor

Su sugrodo cargo

Su sagrodo empeño

Trabajó actuaron muy bonito [linda la perfección de su cargo] Trabajo en lo fiesta alegremente [linda la perfección de su contribución]

Todo to hice mi cargo

[bien completo cumpli mi cargo]

Tudas los hice mi trabajo

Trabaje fuertemente

bo soy trabajo!

Hice todes las cargos

[bo soy contribución]'

estomos wiendo su trabajo

profesendo sus cargos

[estamos viendo su contribución]

Se cumpien sa sogrado cargo

Asi andaremos los horas

[se cumplen su sagrada contribucioin]

670 Te(y) me laj kilbeytik yatel kajwal Nuestro set̃ar alla bo verenos su magnifice abre Laj me kilbeytik spatan kajwal si lo veremos sa corgo de ia fiesto

743 Ya kilbeytikix ya'tel

Laj kilbeytikix spatan yo lo verán su trabajo

Lo winnos sir trabajo abra

lya la vimos su contribución|

(Carnaval de Bachajón, Monod Becquelin, 1973)

La diversidad de los co-textos conlleva una modificación de las traducciones del informador. Pone el acento sobre el hecho de que el trabajo y la contribución del que asume el cargo son en primer lugar aceptar una responsabilidad atribuida por las autoridades (verso 87); luego, considerarla como sagrada (118).

\footnotetext{
"I las traducciones entre corchetes son más o menos literales mientras que la primera es la que fue dada por los tzeltales.
} 
es decir ponerse en condiciones de llenar de belleza y de alegria el ritual ${ }^{17}$ (283); darse "en cuerpo y alma" y volverse uno mismo el sacrificio $\{299\}$ que sólo es valido en presencia de testigos (485). Asi las obligaciones rituales han sido hechas con la perfección requerida: en el verso 492 . Los últimos versos 670-11 y 743-4 describen la periodicidad del tiempo según la cual el pasado alcanza el futuro, elucidando el sentido de este ritual especifico, el carnaval: la continuidad temporal entre la tradición desde el pasado y el abertura hacia el porvenir, el futuro de las comunidades y sus comienzos repetidos.

\section{Disposición de los elementos del paralelismo}

Tanto como la frecuencia, el contenido semántico y las relaciones co-textuales de los elementos en paralelo, su sucesión y su colocación forman parte de las dimensiones del paralelismo. Llamamos "disposición" a los diferentes tipos de asociaciones de elementos del par. Examinemos los diferentes casos que pueden presentarse en un texto. Si el ejemplo precedente presentaba un par fijo (a'tel) patan\} los términos pareados pueden variar a partir de un lexema preeminente \{i.e. localizado en la primera posición del par\}.

\subsection{Emparejaniento fijo ${ }^{1 \mathrm{~s}}$}

El primer tipo concierne a los elementos del par que están siempre ligados. Además se presentan en un orden constante, obedeciendo la mayoria de las veces a la regla del segundo más pesado. Se trata de una formula casi petrificada que va gramaticalizandose (ej. 17). ${ }^{13}$

Los elementos atine,fum "los que se bañan en la tierra" y atine.k'apal "los que se bañan en el polvo" se manifiestan siempre juntos.

\footnotetext{
${ }^{17}$ Quien haya observado, en estos tiempos de fiesta, el suelo de las casas que brilla con las agujas de pino frescas tiradas sobre ell, los altares adomados de flores, los enormes bultos amarillos de maiz en las cocinas, las calabazas y las vasijas cubiertas de telas que esconden sus tesoros destinados a alimentar a los hombres de fiesta, las cajas de refrescos en pilas listas para ser distribuidas, los paquetes de velas blancas como alimento de las santos |... puede entender lo que significz: t'ujbil o'td, t'ujtil patan: meses $y$ a veces àbes de labor para cumplir con ese cargo.

"Para una descripción más detallada y ejemplificada, remitimsos al lector a la tesis de Hull [2003). Su tipologia es muy a menudo similar a la que presentamos aqui. Pares, tripletes, cuadrupletes, quias. mos, elipsis refieren a las mismas formas. Manocalon es equivalente a muestros 'versos aisladas' y llamamos "expansión" lo que describe como "ag mentative parallelism". Las figuras que define como "nesting", "envelopping" y "embedding" forman parte de niveles superiores que consideramos como organizadores del texta más que como disposición de los elementos en paralelo. Por último, analiza. mos aqui el "couplet marking" desde el punto de vista de la frecuencia y no de la semántica.

${ }^{\mathrm{t}}$ No hay consenso a propósito de la defínición de estos sintagmas porçue presentan rasgos tipi. cos de la composición y también una cierta libertad para descomponerse por la menos en tzeltal.
} 
Tzeltal

(30) 472 te wakeb a'tine.lumotik 473 te wakeb a'tine.k'apalotik
Somes las seis que se bañan en fa tierra

Somos las sers que se barian en la basura

(Fiesta de Sun Sebastiản, Monod Becquelin, 1992, recogido en Bachajón).

\subsection{Varios términos "segundos"}

El segundo caso está representado a la inversa por un número más o menos grande de términos en contraste con un mi-par. Como cada elemento del par es polisémico, su sentido varia según las relaciones retóricas que mantiene con el otro componente. Seguin los casos, uno o varios campos semánticos son evocados.

En los discursos rituales tzeltales, al "hijo/a de mujer" se empareja con nich'an "hijo/a de hombre", tehk'an "estar parado, de pie, crecido", untik "descendencia": las asociaciones quedan iguales en el ámbito del parentesco. Lum "tierra" se ve asociado con bahlumilal "mundo", k'apal "polvo, inmundo, basura, falta de", ch'en "gruta, hoyo, lugar sagrado", kaporal "autoridad, hombre, encargado de ritual", ok "pie, masculino, caminante", kaye "camino del pueblo", un conjunto de conceptos que, esta vez, remiten a la geografia, a la organización social, a los géneros, a la toponimia local etc. Esta multiplicidad de asociaciones para un mismo elemento implica relaciones retóricas diferentes (antonimia, sinonimia, metonimia) con otro elemento del par. Este juego de polisemia puede también producirse al nivel del mismo par; en el ejemplo, el informante traducira de manera diferente cada elemento del mismo par según el contexto al cual esté asociado.

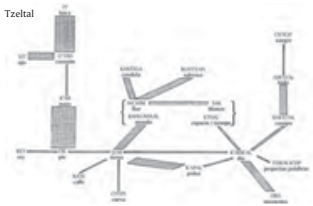

Frousa 7. Rea of asocucaowes de los elementes de pares en un mismo texto 


\section{Configuraciones}

Ya no al nivel del mi-par sino al nivel del par, un cierto tipo de recurrencia lo de no-recurrencia) organiza los pares entre ellos. Es lo que llamamos "configuraciones". Como anteriormente, se distinguen varios tipos de los cuales mencionaremos sỏlo los más simples y los más frecuentes.

\subsection{Configuraciones básicas}

\subsubsection{Paralelismos contiguos}

Se trata del primer nivel de organización de los pares. Es seguramente el lugar en donde las diferencias entre géneros y entre comunidades y lenguas se encuentran más marcadas. En efecto, ciertos textos y gèneros parecen haber rechazado la rigidez acentuada de los paralelismos conexos. He aqui un ejemplo.

\section{Tzeltal}

(3J) 580 Teme xkeluj yu'un

581 Teme xka'buj yu'un

582 Teme ya yawtay kajwal

583 Teme ya sts'uts'uptay kajwal

584 Teme yax ahk'ota yok

585 Teme yax ahk'ota sk'ab 5i los miron

Si los contemplan

Si los griten, sevior nuestro

Si Jos silben, señor nuestro

5i bailan sirs pies

Si bailan surs manes

(Carnaval de Bachajón, Monod Becquelin, 1973)

\subsubsection{Paralelismos en espejo, en quiasmo, en cascadas}

En muchos casos esta conexidad lineal sirve para formar un segundo nivel que se capta si miramos un conjunto más largo: vemos las sucesiones de pares organizándose las unas con las otras, con varios tipos de réplicas que aparecen a primera vista ordenadas arbritrariamente, o revueltas, pero que, en un segundo tiempo, forman conjuntos perfectamente estructurados. Remitimos a un articulo anterior para el inventario de estas figuras (Monod Becquelin, 1993).

Ch'orti'

(32) A1 /tryatravesir /uyok /hyatravesir /uk'ab la fuerza inhibidara de sus pientes

la fuerza inhibidara de sus manos 
B uxek'onir yes /uyatravesir /uyok los dolares de puriolada de lo fuerza

intibidora de sus piernas

uxek'onif yer /hyatravesir /uk'ab' los dolares de puriolada de lo fuerza

intibidora de sus manos

ya'syob' tama e gotera

ahi elibs juegan en los olemos

ya'syob' tama e gotera

ahi ellos juegan en los olerus

B1 uxek'onir yes /hyatravesif /uyok

los dolares de puriolada de lo fuerza

intibidora de sus piernas

uxek'onir yer /hyatravesir /uk'ab' los dolares de puriolada de lo fuerza

inhibidora de sus manos

Muyatravesir /uyok los dolores inhibidores de sur piemas

Takar /Hyatravesir /uk'ab' cont los dolores inhibidares de sus mentos

(Hull, 2002: 7)

\subsection{3. listas, enumeraciones}

Cuando los contrastes evidentes de los propios co-textos son tres, hablamos de "tripletes", entre cuatro de "cuádruples" que están asociados muy a menudo a una disposición en quiasmo — pero a veces la lista se alarga vertiginosamente-. Sin que tengamos grandes argumentos para disociar del paralelismo lo que llamamos "listas" o "series de pares", consideramos no obstante que, aunque se trate de un continuo, puede tratarse de otra configuración.

E primer argumento es que, para tratar de una sucesión de términos articulados en un co-texto común sería arbitrario decidir agruparlos por dos. El segundo argumento, más semántico, ${ }^{20}$ consiste en tomar en cuenta el hecho de que estas "listas" conciernen a campos muy precisos, muy homogéneos y particularmente ligados a la acción ritual. Asimismo como los pares, las listas parecen regidas por un ordenamiento relativamente fuerte y fijo. El ejemplo extremo que damos es extraido de un rezo de curación que ilustra bien este proceso aunque compete a otros tantos procedimientos organizadores.

Tzeltal

(33) 267 jun te ts'anab 268 cheb te ts'anab un espiendor

dos esplendares

${ }^{13}$ Se puda demostrar que las listas presentes en los dialogos rituales provunciados durante el carnaval de Bachajón conciernen únicamente a los duehos del mundo natural, animales y plantas, asi como nombres de santos. Sucede çue este ritual de oposición pone en escena dos grupos anta. gonistas, que son las autoridades de la comunidad y los representantes del munda de la seha, cuya reunión ritual produciri el renacimiento de la comunidad (Monod Becquelin y Breton, 2003). 
269 oxeb te ts'anab

270 chaneb te ts'anab

271 jo'eb te ts'anab

272 wakeb te ts'anab

273 hukeb te ts'anab

274 waxakeb te ts'anab

275 baltuneb te ts'anab

276 lajuneb te ts'anab

277 bulucheb te ts'anab

278 lajchayeb te ts'anab

279 oxlajuneb te ts'anab

280 jun te ts'akun te

281 cheb te ts'akun te

282 oxeb te ts'akun te

283 chaneb te ts'aktun te

284 jo'eb te ts'akun te

285 wakeb te ts'akun te

286 hukeb te ts'akun te

287 waxakeb te ts'akun te

288 baluneb te ts'akun te

289 lajuneb te ts'akun te

290 bulucheb te ts'akun te

291 lajchayeb te ts akun te

292 oxlajuneb te ts'akun te

293 jun te ts'akun te chi

294 cheb te ts'akun te chi

295 oxeb te ts'akun te ch

296 chaneb te ts'akun te chi

297 jo'eb te ts akun te chi

298 wakeb te ts'akun te chi

299 hukeb te ts'akun te chi

300 waxakeb te ts'akun te chi

301 baluneb te ts'akun te chi

302 lajuneb te ts'akun te chi

303 bulucheb te ts'akun te chi

304 lajchayeb te ts akun te chi

305 oxlajuneb te ts'akun te chi

306 jun te ts'akun te bak

$[\ldots]$

318 oxlajuneb te ts'akun te bak tres espiendores

cuatno esplendares

cinco esplendores

seis esplendores

siete espiendares

ocho esplendares

nueve esplendores

diez esplendoves

ance esplendares

doce espiendares

trece espiendores

una uniton

des unianes

tres uniones

cuatro uniones

cinco unianes

seis uniones

siete untiontes

ocho uniones

nueve unjones

diez uniones

ance uniantes

doce untanes

trece unianes

una uniton de fa vena

dos unianes de lo veria

tres uniones de la vena

cuatro untiones de la vena

cinco uniantes de la vena

seis uniones de la vena

siete urtiontes de lo vena

ocho wiones de fa veno

aweve uniones de la vena

diez uniones de la vena

ance untantes de la vena

doce unianes de lo vena

trece uribanes de la wena

una unión del hueso

trece unianes del hueso 
319 jun te ts'anab

$[\ldots]$

331 oxlajuneb te ts'anab

332 jun te cir'uht

|...

344 axlojurieb te chinht

332 jun te pulaw

$[$....

357 axiojuneb te pulaw

358 jun te ts'akun te nujk'ul

$[\ldots]$

370 axlojuneb te ts akun te mujk't? un esplendor

trece esplendores

un estónago

trece estómagas

un $7 ? ?$

trece ???

una uniton de Ia pjel

trece untones de la piel

"Mais jai transmí fesperance", Breton y Monod Becquelin, 1989)

\subsection{Configuraciones complejes}

Aun cuando la estructura no se ve de inmediato, permanece una organización en varios niveles. Una presentación de este corto extracto del Chilam Balan de Chumayel lo atestigua. Veremos que para cada columna aparecen paralelismos, a excepción de la última. En la primera columna se pueden apreciar las expresiones de movimiento, en la segunda los topónimos más o menos bajo la forma de una lista, en la tercera las descripciones en par, y en la última los nombramientos.

\section{Maya yucateco colonial}

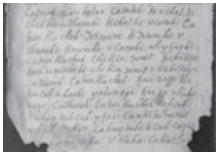

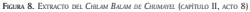



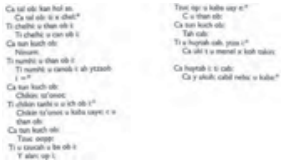

Ficuan 9. PResentación por Eostonson (1986) del mismo extractio

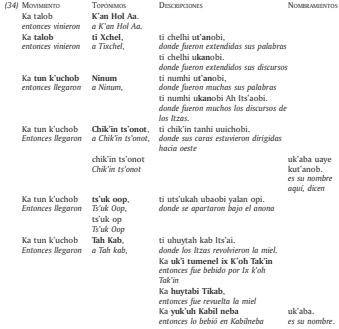

(Capitulo II del Chilam Balam de Chumevel, Edmonson led.f. 1986) 
Bright, en el articulo ya mencionado sobre el difrasismo, ha estudiado la organización del capitulo VII de los coloquios y doctrina cristiana editados y comentados por León-Portilla (1986). Conduye que la organización del texto sigue dos principios: binarismo (binariness) y encajonamiento (enbedding) (Bright, 1990: 450). Pero su preocupación era principalmente la de identificar una versificación y su análisis queda subordinado al binarismo.

\subsubsection{El estatuto de la frecuencia}

Otro nivel de configuración puede ser apreciado por el examen de la frecuencia de los pares y sus posiciones respectivas.

La frecuencia alta de los pares ha sido estudiada en tzotzil y tzeltal y relacionada con el "calor", la fuerza y el suceso del discurso (Gossen, 1974b). Pero el estudio estadistico sobre la totalidad del Rabinal Adhi (cfr. Breton, 1999) hace resaltar una situación tal vez más compleja, donde se distinguen claramente pares muy frecuentes y otros, numerosisimos, que aparecen una sola vez.

Reparto de los pares en el Rabinal Achi:

- Número de líneas: 2599

- Número de pares: 1048

- Número de mi-pares: 2096 , o sea, $80.6 \%$ de las lineas son paralelísticas.

En primer lugar, este histograma muestra la omnipresencia del fenómeno del paralelismo en el texto.

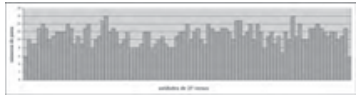

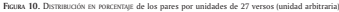

La gráfica siguiente muestra que los pares que aparecen sólo de una a cuatro veces en el conjunto del Rabinal Achi (entre más de 2500 versos) representan más de $3 / 4$ de las ocurrencias en el texto.

Por fin el cuadro 3 muestra los pares más frecuentes, es decir los que representan más del $1 \%$ de todas las ocurrencias de pares del conjunto del Rabinal Achi. La relación entre la frecuencia de un par, su importancia semántica y su estatuto funcional en el texto depende de numerosos factores. No obstante, pareceria que los pares más recurrentes dan la trama general al documento tratado (Monod Becquelin, 1987). En el Rabinal Achi, los pares más frecuentes son kaj/ulew 


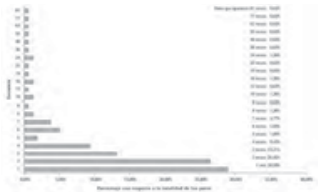

Foguka 11. Poecentaye of fakes por nümero de ocurrencias

"cielo/tierra", $t z$ 'aq/k'oxtun "fortaleza/muralla", juyub/toq"aj "montaña/valle", oyew/ achi "hombre colérico/hombre verdadero", ajow/winaq señon/hombre eminente, ch'/wach "boca/rostro", alik'ajol "hijo de mujer/hijo de hombre" y son indices fuertes del contenido del texto (drama politico en relación con el antagonismo territorial entre Rabinal Achi y K'iche' Achî. Los temas y las circunstancias contextuales están tomados en cuenta por la disposición de las variantes en el curso del texto y las distancias entre los bloques repetidos, muchas veces separados por la presencia de versos o de lineas aisladas, así como por otros procedimientos. Para evaluar esta hipótesis de lectura, remitimos a la obra misma.

Cunaso 3. Rersingón ex necextajes de los pares que representan mis del 12 del conjunto de pares

\begin{tabular}{|c|c|c|}
\hline & & 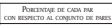 \\
\hline $\operatorname{kaj} / \mathrm{di}$ iew & "cielo / tierra" & $15.36 \mathrm{~s}$ \\
\hline tz'aq ik'axlun & "Fortaleza / muralla" & $7.73 \%$ \\
\hline juyub / tog'aj & "montaña / valle" & $735 \%$ \\
\hline onew / achi & "hombre colérico / hombre verdadero" & $5.92 \%$ \\
\hline ajaw / winaq & "señor/ hombre eminente" & $5.25 \%$ \\
\hline dir'/ math & "boca / rostro" & $3.82 \%$ \\
\hline ail/k'ajol & "hijo de mtujer / hijo de hombre" & 3.448 \\
\hline yebal/sik'ibal & "desafio / grito" & 2.298 \\
\hline ch'ab/ podob & "arco / escudo" & $2.29 \%$ \\
\hline uq'alel / rabinal & "dignatario / Rabinal" & $2,10 \%$ \\
\hline $\operatorname{lnam} / \operatorname{soch}$ & "morir/perderse" & 1.818 \\
\hline hot / balam & "Siguila / jaguar" & $153 \%$ \\
\hline aj uxab / aj pop̣'amab & "Ios Uxab/los poq"omab"' & $15,3 \%$ \\
\hline wixal / kutami? & "raiz/tronco" & $1.15 \%$ \\
\hline
\end{tabular}


A fin de comprobar esta hipótesis, examinemos en otros tipos de textos la relación entre la frecuencia y la trama. Ciertamente, este lazo es previsible puesto que la forma de la insistencia en el texto está ligada al tema del discurso. No obstante, los mayas no se contentan de esta validación del tema por la frecuencia, además dan varias versiones de él. Para una boda, por ejemplo, habrá varios cuadros posibles.

Asi, un conjunto de pares recurrentes y un tema son interdependientes, un mismo par en dos conjuntos diferentes tendrá una interpretación distinta: $t e^{\prime}$ / chajan en un discurso de cambio de cargo tendrá la significación de "autoridad mayor/autoridad menor" a diferencia de los ejemplos siguientes.

Estos ejemplos son un análisis de tres discursos de pedidas de novia y de un rezo de bendición de milpa hechos por un especialista ritual. Vemos que las orientaciones de cada uno de los discursos difieren ligeramente.

El cuadro 4 presenta los pares más frecuentes (con numero de ocurrencias superior a dos) en transcripciones de performances orales donde el fenómeno del paralelismo está muy presente. Si una gran parte de los pares están presentes en cada una de estos performances, podemos notar igualmente que un par como allnich'an "hijo de mujerhijo de hombre" tiene un estatuto muy importante en términos de ocurrencias en los textos Tze04, Tze05 y Tze06, que corresponden a las tres pedidas de novia sucesivas y esta ausente del texto Tze 18 (bendición de la milpa). En este último texto, pares relativos al territorio como lum/bahlumilal "suelo/tierra", $k$ "inalibahlumilal "espacio/tierra", pot/kuhk "espalda/esquina" o ik'alitokal "viento/nube" tienen un estatuto predominante. En lo que concieme el "montaje" de los tres textos de pedidas de novia, el primero insiste en la complementariedad - te/chajon evocando el ärbol y la liana, asi como las figuras del hombre y de la mujer- mientras que el segundo tiene que ver sobre todo con la unión que resulta del matrimonio de tales entidades complementarias y el tercero con la fundación de un hogar y la identidad de los lugares que derivan de ésta. 
Cuvano 4. Distkancoón de lós muses por numero de ocurrencias en cuatro textos

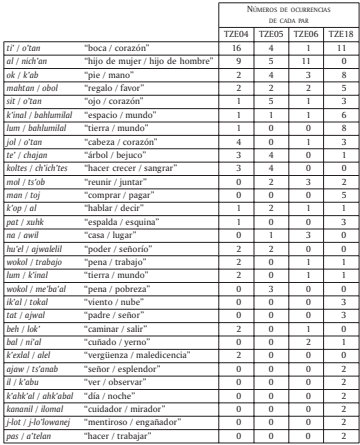

\subsubsection{Los elementos demarcativos}

Nos falta hablar de herramientas que calificamos de demarcativas, por delimitar bloques de significación: el sistema paralelístico está entrecortado con versos/ 
lineas aislados y förmulas invocatorias, ${ }^{21}$ que permiten un respiro, un cambio. una dinámica, que inauguran otra parte y solicitan la memoria (Brody, 1986: 268-269).

\section{Tzeltal}

(35) 33 pero yo'tika tate jnich'(a)ajel

34 pero bin k'an ku(t)ilika stukel

35 stojol yahl jkuxlejaltik

36 stojol yahl jtehlumaltik

37 teme ya jmajantikix te mesa

38 teme ya jmajantikix te banko

39 teme ya kai'ytikix jayeb ora

40 teme ya kat'ytikix jayeb Kahkal

41 but'il ya (t)saki sbah jti'tik

42 but'il ya (t)saki sbah ko'tantik

43 ora yo'tika tate jnich'(a)najel

44 tey me jmajanbetik sti'

45 tey me jmajanbetik yo tan

46 te yahl escribano tatil

47 te yahl escribano ajwalil y ahora padre que nos engendra

pero que podemos decir

ef precio de muestras vidos

ef precio de nuestres lugares de existencia

yo prestamos la mesa

ya prestamos el banco

yu sobemos aquí cuántas haras

yu sabemos aqui cuáras dias

camo se unen nuestras bocas

como se unen nuestros carazones

y ahora padre que nos engendra

ahi donde le prestamos lo boca

ahi dande le prestamos el carazain

del padre escribano

del escribano seriot

(Fiesta de San Sebastiain, Monod Becquelin, 1992, recogido en Bachajón)

La delimitación de estos bloques puede igualmente apoyarse sobre la recurrencia de secuencias idénticas de pares que juegan un rol útil de demarcación, además de su valor propio significativo (ej. 36); esta dimensión organizadora utiliza un conjunto entero de paralelismos, que escinde el texto; los conjuntos de pares se fijan, lo que era variante se vuelve invariante.

Estos cambios de función afectan la percepción auditiva y llaman sin cesar a la recomposición de lo que se ha dicho a partir de lo que se dice.

Achi de Rabinal

qatz k'u are lal kamel

lal sachel

2025 waral ch(i) uxmut kaj

chil uxmut ulew

\author{
iEs seguro que wais a morir \\ que wais a desaparecer \\ aquil, en el ombligo del cielo \\ en el ambligo de lo tierra!
}

$|\cdots|$

${ }^{21}$ Na contamos con espacio suficiente para dar un ejemplo entero, pero de manera general para los discursos rituales de Bachajón, estas fórmulas de referenciaimvocación, que se cuentan entre las más frecuentes, pueden ser mucho mis largas, delimitando bloques cada vez más cortos a medida que se acerca el final del discursa. 
2537 ajkaroq kaj ajkaroq ulew

la qatzij waral in kamel in sachel

waral ch(i) uxmut kaj ch|it uxmut ulew

[...

2570 ajkaroq ba la kaj ajkaroq ba la ulew we qatz waral in kamel

in sachel

waral ch(i) uxmut kaj ch/a) uxmut ulew joh cielo.

Oh therra!

Eis cierto que agui marire

desaparecere,

aqui, en el autbligo del cielo

en el anbligo de lo tierna?

"Joh Ves, cielo

oh Vis, tierrat

Si verdaderumente miers nộ desaparezo, aqui, en el aubbīgo del cielo.

en el aubligo de lo tierra...

(Robival Ash, Breton, 1999: 261, 289, 290)

Estas herramientas significativas y demarcativas delimitan unidades de todo tamaño: proposiciones y macro-proposiciones, secuencias y macro-secuencias, episodios, ciclos, "chunks", según las terminologias adaptadas a diversas orientaciones de investigación (Guézennec, s. f.). Lo que nos interesará es el análisis de las distancias entre las unidades asi separadas, necesario para la comprensión de la estructura formal y pragmática del texto. En efecto los niveles de integración diferentes de los componentes del texto se construyen gracias a estos ecos que pautan las olas de palabras y atizan la memoria.

\section{Sobre las funciones, las intenciones y las palabras}

Falta aún considerar $\mathrm{e}$ texto en su totalidad $\mathrm{y}$ su intertextualidad: sólo estos niveles permiten descifrar verdaderamente el sentido manifiesto y las inferencias que le dan toda su significación. Particularmente visible en la evocación de asociaciones virtuales que dan sentido a un par además de su valor local, el mismo mecanismo se produce, como en todo el texto que se edifica sobre procedimientos paralelisticos.

La construcción de un cuento o de una plegaria, definida por su organización paralelistica, evoca inmediatamente, en el espiritu de los auditores, construcciones similares y conjuntos significativos. En un programa próximo, nos detendremos a definir, tanto como sea posible, los diferentes géneros que se encuentran en la tradición oral, géneros que imponen/reciben una disposición y una configuración del paralelismo caso por caso. ${ }^{2}$

${ }^{22}$ Brody (1986) asocia a unz tipologia de los géneros los uses diferentes de la repetición y del paralelismo. 
Giertamente la definición del género es una parte esencial de lo que será la significación. Es en el seno de un género que se fijan las variaciones del locutor, incluyendo el hecho de deshacer las reglas, y mezclandolas con otros géneros a su voluntad. Hemos notado que en un caso de rezo de cura, la finalidad de ésta impone a la vez trayectoria - el recorrido de los lugares donde la enfermedad ha podido advenir-y una multidireccionalidad de puntos de vista a partir de la búsqueda de la etiologia de la enfermedad, la personalidad del enfermo y la biografia del curandero: tantos parämetros fundamentales que proyectan una amplia cantidad de posibles sobre la terapia (véase Monod Becquelin y Breton, 1989).

En el ejemplo siguiente (37), esta bisqueda del lugar de "contaminación" por un espiritu malo empieza a través de elementos asociados a los lugares de ofrenda a la tierra (aquí personificada por Santa/ doña Magalena del Mundo). En un segundo tiempo, en la parte que contiene el par ok/k'ab' "pie/mano", se señala la localización en el momento del ritual de esta enfermedad, es decir en el cuerpo del enfermo. Por último, la búsqueda sigue con elementos asociados a la casa y a los lugares de ofrendas domésticas. Esto va en el sentido de Rumsey (2001) que ve en la utilización del paralelismo una capacidad de unir mimesis y diégesis o como lo dice èl: "el mundo del enunciador y el de la narración", lo que llama él "meta-narracionn".

\section{Ch'orti'}

(37) a asi tamat enyax mesa tamar e mesa antibano tamar e mesa anterior tamar e mesa olvidado a'asi tamat enyax kaxtiren ensak kaxtiren a'asi tamar enyax chumpi' ensak chumpi' entonses yaha? a'asi tamar enyax jolote ensak jolote entonses yaha' pues ya' ch'a't e donya magalena del mundo
santa magalena del mundo ch'a'r takat uremolino yer uyok takar uremolino yer uk'ab" ch'a't takar ulat'ahner uyok takar ulat'ahner uk'ab' takar utihb yanis uyok takar utihb'yanir ukab'
Ét juega en la mesa vende (altar) ent fa mesa "antibano" (oltar de los antignos) ent lo meso "onteriar" (alter de fes entiguas) ent lo meso "olvidodo" (aitor de los antigasos) É juega en la galina verde lo gollina blance

El juega en el pavo verde entonces afil pano bianco

Ef juega en el pavo verde entonices ofti pues pavo blanco

Ahí está donia Magalena del Mundo Santa Magalena del Mundo el está can el torbelinho de sus pies cant ef remoline de sus manos Ê se encuentra con la catatonia de sus pies con la catatonia de sus mentes con ef temblor de sus pies con el temblor de sus manos 
ch'a't che takar

entonses ya' ch'a'ar

a'ass tamar enyax gotera

ensak gotera

a'asi takar e don juan de portero

donya juana de portero

entonses yaha

ya' ch'a'ar a'asi ta enyax meson

ensak meson

atasi tamar enyax petición

tamat ensak peticion
E) Se encuentra con

entonices el se encuentra ahi

El juega en la conolera verde

fa canaleta bianco

Ej juega con Don fuant de portero

entouces ahi

dima juena de portero

É se encuentra ofit jugando en el aitor verde

ett el blanco altar

Et juega en la ofrenda verde

en la ofrenda blanta

(Rezo de curación, Becquey, 2007, recogido en Oken)

En un cuento, otras finalidades pueden estar presentes. La estructura, menos compleja - "the couplet structure of myth is looser and less consistent" dice Victoria Bricker (1974: 375 ) - permite adivinar otro tipo de objetivo narrativo. muy alejado por cierto del cuento moralizador occidental. Si observamos un ejemplo tzeltal, el cuento de la milpa del sol, constatamos que entre los pasajes demarcativos que dan ritmo al relato $(A)$; se inscriben episodios $(1,2 \ldots)$ -variantes estructurales del texto- pero que estas variantes cambian entre ellas mismas según criterios enunciados más arriba: las preocupaciones de cada narrador con respecto a una plaga agricola sufrida por la comunidad, le incitarán a introducir en esta perspectiva los elementos variables dentro de la estructura que toman entonces figura de variaciones. Al pájaro (episodio 1, cuento 1) se sustituirán los cérvidos (episodio 1, cuento 2). A los aguties que siguen al pájaro en la versión anterior, los remplazarán los roedores. La finalidad de este cuento puede ser asimilada a una crónica, género todavia mal definido pero que parece de gran importancia en la tradición maya contemporanea, como lo es en otras tradiciones indigenas. 
Tzeltal

\begin{tabular}{|c|c|}
\hline 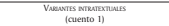 & 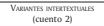 \\
\hline $\begin{array}{l}\text { A } \\
\text { ya la xtal yil ts'ihin te snane } \\
\text { se dice que su mame viene para ver } \\
\text { ja' ts'ihin tawaye ya la yil ts'ihin } \\
\text { y lo que ve enseguida, se dice } \\
\text { jahchemix te' xchakola } \\
\text { es que los áboles se han feventodo de nuevo }\end{array}$ & $A^{\prime}$ \\
\hline Episodio i del nupao xemain & Episodio 1 de los cérusas \\
\hline $\begin{array}{c}\text { A } \\
\text { ya ta yil ts'thin } \\
\text { se dice que ella ve enseguida } \\
\text { jahchemix te' xchakola; } \\
\text { a fos érboies levantandose de nuevo }\end{array}$ & $A^{\prime}$ \\
\hline Episodio del acieni & $\begin{array}{l}\text { Episodio de los noebones } \\
\text { (ratas, conejos, etc) }\end{array}$ \\
\hline A & $A^{\prime}$ \\
\hline $\begin{array}{c}\text { ya ta yil ts'thin } \\
\text { se dice que ella ve enseguida } \\
\text { jahchemix te' xchakola; } \\
\text { a fos érboles levantandose de nuevo } \\
\text { etc. }\end{array}$ & \\
\hline
\end{tabular}

De esta forma, el objetivo de la próxima investigación será entender cómo se articula este doble procedimiento de diferenciación y de similitud con las funciones de los textos y las intenciones de los locutores. El articulo de Vapnarsky (en este volumen) lo ilustra perfectamente.

No podemos más que abrir aún las perspectivas que ofrece el paralelismo, este procedimiento de "memoria del futuro" - segun la expresión de una historiadora de los mayas (Farriss, 1987)—, herramienta cogniscitivativa, teórica y poética.

E paralelismo combina varias visiones del universo. El locutor escoge en la profusión de emparejamientos virtuales, lo que quiere resaltar, eligiendo la figura pertinente (ya sea descriptiva, metafórica o sorprendente...). Esta evoca, dibuja, manifiesta, hace visible el universo cotidiano, onirico, ritual, etc., y abre las fronteras entre las categorias. Un par puede unir mimesis y diégesis, el locutor y 
el oyente (curandero/santo), en tanto que mundos reconciliados. Además, y éste no es uno de sus méritos menores, tiene una función mnemotécnica colectiva esencial.

Por último, es una transmisión de lo bello. Paul Valëry escribía: "no soy un poeta, soy un versificador". Para nuestros ojos, los mayas podrian decirse "poetas Y versificadores", si tomamos el término como lo concebia el autor: sonido antes que nada. En este conjunto de procedimientos se despliegan la música, la mètrica, la medida, el énfasis, el orden. Los auditores-espectadores, activos por su rol de testigos, y que constituyen el público de actos rituales, pero también el de los cuentos, de los testimonios de sueños o de narraciones de la historia, esperan las "palabras adornadas", "florecidas", "elegantes" que los mayas consideran como el alimento de los dioses, de los santos y de los dueños de los espacios. ${ }^{23}$ Para ser bellas, éstas tienen que ser "adecuadas", es decir tienen que obedecer a una función ética y estética, esencial para los indios de norte a sur del continente cuando se trata del Verbo.

\section{BIBLIOGRAFIA}

Becker, Alton L.

1984 "The linguistics of Particularity: Interpreting Superordination in a Javanese Text", Berkeley Lingurstic Society, 10: 425-436.

Bolles, David, y Alejandra Bolles

1996 A Grantnor of the Kontecan Mayan Language, revised edition. New Hampshire: Labyrinthos Press.

Breton, Alain

1999 Robinal Achi. Un drama dinóstico maya del sigfo xv. Guatemala: CEMCA.

- y Aurore Monod Becquelin

1989 "Mais j'ar transmis lesperance...". Etude d'une priere de guerison tzeital Maya du Chiopos, Mexięue), Chantiers Amerindia 13, Amerindia. Paris: AEA.

Bricker, Victoria $\mathrm{R}$.

1974 "The Ethnographic Context of Some Traditional Mayan Speech Genres". Expiowations in the Ethnography of Speaking, pp. 368-389, R. Bauman y J. E. Sherzer (eds.). Cambridge: Cambridge University Press.

s. f. The Function of Parallelism in Tzotzil Narrative.

\footnotetext{
2. "Los principios de la orgarización que producen una poetica medida, y la aparición de expectativas frente a la estructura. Ideas coordinadas se ordenan en sintagmas, oraciones y párrafos que equilibran un elemento con otro de similar importancia y forma. La repetición de los sonidos signifi. cados y estructuras sirve para ordenar, poner énfasis y apuntar a ciertas relaciones" (Rumsex, 2001 ).
} 
Bricker, Victoria R., y Munro S. Edmonson leds.)

1985 "Supplement" to the Hondbook of Middle American lidians, vol. 3, Literafures. Austin: University of Texas Press.

Bright William

1990 "'With One Lip, with Two Lips': Parallelism in Nahuatl", Language, 66 (3): $437-452$.

Brody. jill

1986 "Repetition as a Rhetorical and Conversational Device in Tojolabal (Mayan)", Internotional foumal of Americen Linguistics, 52: 255-274.

Cuevas Cob, Briceida

2007 "Poèmes", fointure, 85: 2-31.

Damell, Regna

2003 "Dell Hymes, Now I Know Only So Fur: Essays in Ethnopoetics", reseña de.... Anthropological Linguistics, 48 (1): 823 .

Derrida, Jacques

1976 of Grammatology. Baltimore: Johns Hopkins University Press.

Edmonson, Munro S.

1986 Heoven Born Merido and its Desting. The Book of Chilam Balom of Chumayel. Austin: Lniversity of Texas Press.

Farriss, Nancy

1987 "Remembering the Future, Anticipating the Past: History, Time and Cosmology among the Maya of Yucatán". Comparotive Studhes in Sociery and Histary. 29: $566-593$.

Fox, James J.

1974

"Our Ancestors Spoke in Pairs: Rotinese Views of language, Dialect and Code", Expiorations in the Ethnography of Speaking, pp. 65-85, R. Bauman y J. E. Sherzer (eds.). Londres: Cambridge University Press.

1975 "On Binary Categories and Primary Symbols: Some Rotinese Perspectives". The Interpretation of Symbolism, pp. 99-132, R. Willis (ed.). Londres: Wiley.

1988 To Speak in Pairs: Essays on the Ritwol Langanges of Eastem Indonesia. Cambridge: Cambridge Liniversity Press.

Garibay Quintana, Ángel Maria

1953 Historia de la literatura nóluatl, primera parte. México: Porrúa.

Goody, Jack

1979 La raison graphique: la damesticotion de la pentsé samoge. Paris: Éditions de Minuit. 
1994 Entre l'orolite et l'écriture. Paris: PUE:

Gossen, Gary H.

1974a Chemules in the World of the Sun: Time and Space in a Mayo Orol Tradition. Cambridge: Harvard University Press.

1974b "Toward an Ethnology of Speaking: Introduction", Explorations in the Ethnography of Speoking. R. Bauman y J. E. Sherzer (eds.). Cambridge: Cambridge University Press.

2002 Four Creations: An Epic Story of the Chiopos Moyas. Norman: University of Oklahoma Press (The Civilization of the American Indian Series).

Grimes, Joseph E.

1972 "Outlines and Overlays", Language, 48 (3): 513-524.

Guézennec, Nathalie

s. f. Les paralletismes comme thode de counposition ovale des contes? Êtude de quelques contes bos-breton. Manuscrito.

Halliday, Michael A. K.

1967 "Notes on Transitivity and Theme in English $\langle\mathrm{II})^{\circ}$, journal of Linguistics 3 (2): $199-244$.

Hanks, William F.

1999 Intertexts: Witings on Language, Utterance, and Comtext. Lanham. Maryland: Rowman \& Littlefield Pub.

Haviland, John B.

1992 "Lenguaje titual sin ritual", Estudios de Cuitura Moyo, 19: 427-443. México: UNAM.

Hull, Kerry

2000 "Cosmological and Ritual Language in Ch'orti". Foundation for the Advancement of Mesoametican Studies (http/Www.famsi.org/reports/99036) index.html.

2002 "A Comparative Analysis of Chorti" Verbal Art and the Poetic Discourse Structures of Maya Hieroglyphic Writing". Foundation for the Advancement of Mesoamerican Studies (http//www.famsi.otgreports/00048/index.html).

2003 'Verbal Art and Performance in Ch'orti' and Maya Hieroglyphic Writing". Tesis doctoral, The University of Texas at Austin.

Hymes, Dell

2003 Now I Know Only So Far: Essays in Ethnopoetics. Lincoln: University of Nebraska Press. 
Jakobson, Roman

1973 Questions de poétique. Paris: Le Seuil.

Kerbrat-Orecchioni, Catherine, y Véronique Traverso

2004 "Types d'interactions et genres de l'oral", Langages, 153: 41-51 (Les genres de la parolel.

Lacadena, Alfonso

"Aptuntes para un estudio sobre literatura maya antigua", Texto y comtexto: Perspectivas intracuiturales en el análisis de lo literatura mavo yecoteca, A. Giansenheimer, T. Okoshi Harada y J. F. Chuchiak (eds.). Bonn: BAS (en prensa).

Laughlin, Robert M.

1980 Of Shoes and Ships and Sealing Wax, Sundries from Zinatantan. Washington, D.C.: Smithsonian Institution Press.

Launey, Miched

1992 Introducción a la lenguo y a fa lireratura náhuatl. México: UNAM.

Lounsbury, Floyd G.

$1980 \quad$ "Some Problems in the Interpretation of the Mythological Portion of the Hieroglyphic Text of the Temple of the Cross at Palenque", The Third Palenque Round Table, 1978, V (2): 99-115. M. Greene Robertson (ed.). AustinLondres: University of Texas Press.

León-Portilla, Migsel (ed.)

1986 Cologuios y doctrina cristiane, cart que las doce froiles de San francisco, enviodos por el popa Adrieno $1 /$ y por el emperador Corios $V$ convirtieran a los indios de ia Nueva Esporia. Ent Jengua mexicana y esporiala. Edición facsimilar, introducción, paleografía, versión del náhuati y notas de Miguej León-Portilla. México: UNAM.

Monod Becquelin, Aurore

1987 "Le tour du monde en quelques couplets. Le parallélisme dans la tradition orale maya", Kolevoío et traditions orales du monde, pp. 467-488. J. FernandezVest (ed.). París: Editions du CNRS (Colloques internationaux).

1993 "Monologues polyphoniques. Les discours rituels maya", Pour une anthropologie des woix, pp. 295-329, N. Revel y D. Rey-Hulman (eds.j. Paris: LHarmattan.

1995 "Formes et figures dans les premiers sermons tzeltal, Amerindia, 19-20: 53-95 fnutmero especial: La découverte des langues et des écritures d'Amérique).

2006 "E Jenguaje sagrado contemporáneo. Comentarios sobre la definición de los géneros en la tradición oral maya contemporánea", Socred Books, Socred Languages. Two Thousand Vears of Ritual and Religious Mayo Literature. Proceedings of the 8th European Mayn Conference (Modrid, November 25-30, 2003), pp. 
173-199, Acta Mesoamericana, R. Valencia Rivera y G. LeFort (eds.). Markt Schwaben: Verlag Anton Saurwein.

\section{y Alain Breton}

2002 La guerre nouge ou une politique maya du sacre. Un carnaval tzeltal au Chiapas, Mexique, París: CNRS Editions.

2003 "¿Cuál espacio para los kabinal de Bachajon?", Espacios mayas: usos, representtaciones, creencios. pp. 327-362. A. Breton, A. Monod Becquelin y M. H. Ruz (eds.). México: UNAM, Centro de Estudios Mayas/CEMCA.

Montes de Oca Vega, Mercedes

1997 T.os difrasismos en el náhuat, un problema de traducción o de conceptualización". Amerindia, 22: 31-46 (Ttaducción y Alteridad Linguística, tomo 1).

Norman, William $\mathrm{M}$.

1980 "Grammatical Parallelism in Quiché Ritual Language", Mrocedings of the Sixth Antud Meeting of the Berkeley Linguistics Society, pp. 387-399. Berkeley: Berkeley Linguistics Society.

Rumsey, Alan

2001 'Tom Yaya Range: A Metrical Narrative Genre from the New Guinea Highlands", Journal of Linguistic Anthropology, 11 [2]: 193-239.

Ruz. Mario H. (ed.)

1989 Las lenguas del Chiapas colotial. México: LNAM.

Sharet, Robert ). (en colaboración con Loa P. Traxler)

2006 The Aritient Moyo, saxth edition. Stanford: Stanford University Press.

Stuart, David

2003 "On the Paired Variants of TZ'AK", Mesoweb thtrp:/Www.mesoweb.comistuart/notesizak.pdf).

Tatunen, Deboraht

1987 "Repetition in Conversation: Toward a Poetic of Talk", Longuage, 63 (3): 574605 .

1989 "Talking Voices: Repetition, Dialogue and Imagery", Conversationel Discourse. Cambridge: Cambridge University Press.

Vapnarsky, Valentitua

1999 Conceptions et expressions du temps chez les Mayas Yucatèques fQuintana Roof $)^{*}$. Tesis doctoral, Université Paris X-Nanterre.

2008 "Paralelismo, ciclicidad y creatividad en el arte verbal maya yucateco", Estudios de Cuitura Mayo, 32: 151-196. México: UNAM, Instituto de Irvestigaciones Filológicas, Centro de Estudios Mayas. 